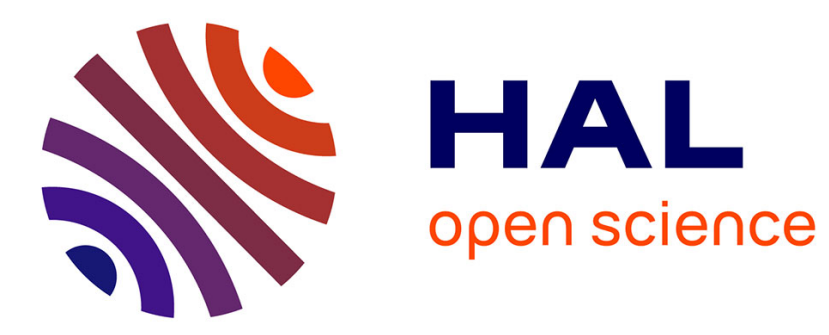

\title{
Dynamic context logic
}

Guillaume Aucher, Davide Grossi, Andreas Herzig, Emiliano Lorini

\section{To cite this version:}

Guillaume Aucher, Davide Grossi, Andreas Herzig, Emiliano Lorini. Dynamic context logic. Second International Workshop on Logic, Rationality, and Interaction (LORI 2009), Oct 2009, Chongqing, China. pp.15-26, 10.1007/978-3-642-04893-7_2 . inria-00556156

\section{HAL Id: inria-00556156 \\ https://inria.hal.science/inria-00556156}

Submitted on 8 Sep 2013

HAL is a multi-disciplinary open access archive for the deposit and dissemination of scientific research documents, whether they are published or not. The documents may come from teaching and research institutions in France or abroad, or from public or private research centers.
L'archive ouverte pluridisciplinaire HAL, est destinée au dépôt et à la diffusion de documents scientifiques de niveau recherche, publiés ou non, émanant des établissements d'enseignement et de recherche français ou étrangers, des laboratoires publics ou privés. 


\title{
Dynamic Context Logic
}

\author{
Guillaume Aucher ${ }^{1}$, Davide Grossi ${ }^{2}$, Andreas Herzig ${ }^{3}$, and Emiliano Lorini ${ }^{3}$ \\ ${ }^{1}$ University of Luxembourg, ICR \\ guillaume.aucher@uni.lu \\ ${ }^{2}$ University of Amsterdam, ILLC \\ a.grossi@uva.nl \\ 3 University of Toulouse, IRIT \\ \{herzig, lorini\}@irit.fr
}

\begin{abstract}
Building on a simple modal logic of context, the paper presents a dynamic logic characterizing operations of contraction and expansion on theories. We investigate the mathematical properties of the logic, and show how it can capture some aspects of the dynamics of normative systems once they are viewed as logical theories.
\end{abstract}

\section{Introduction}

In artificial intelligence as well as in philosophy, contexts - when addressed formallyare often thought of as sets of models of given theories [7] or, more simply, as sets of possible worlds [17]. Once a context is viewed as a set of models/possible worlds, its content is nothing but the set of logical formulae it validates or, otherwise, its theory or intension.

This perspective has been used, in [9], to develop a simple modal logic for representing and reasoning about contexts. This logic is based on a set of modal operators $[X]$ where $X$ is a label denoting the context of a theory. A formula $[X] \varphi$ reads 'in the context of $X$ it is the case that $\varphi^{\prime}$. The present paper develops a 'dynamification' of such logic by studying the two following operations:

- Context expansion (in symbols $X+\psi$ ), by means of which a context is restricted and hence, its intension-its logical theory—strengthened. Such operation is similar to the operation for announcement studied in DEL [21]. Its function is to restrict the space of possible worlds accepted by the context $X$ to the worlds where $\psi$ is true.

- Context contraction (in symbols $X-\psi$ ), by means of which a context is enlarged giving rise to a weaker logical theory. The function of this operation is to add to the space of possible worlds accepted by context $X$ some worlds in which $\psi$ is false.

The resulting dynamic logic is studied from the point of view of its mathematical properties and illustrated through a running example. Just like the context logic introduced in [9] was developed to cope with some problems in the analysis of normative systems, its dynamic version will be illustrated by resorting to examples taken from the normative domain. In particular, we will link context expansion to some kind of "promulgation" of norms and context contraction to some kind of "derogation" of norms 1

\footnotetext{
${ }^{1}$ The terms promulgation and derogation are borrowed from the first paper addressing norm dynamics by formal means, that is, [2].
} 
This illustration will highlight the features that norm dynamics shares with theory dynamics once norms are viewed as logical statements of the type: "a certain fact $\varphi$ implies a violation". Such view of norms builds on those approaches to deontic logic which stemmed from the work of Anderson [4] and Kanger [12]. Although rather abstract and, under many respects, simplistic such view of norms has received considerable attention by recent developments in the logical analysis of normative systems within the artificial intelligence community (e.g., [1, 13, 18]).

The paper is organized as follows. In Section 2 we will briefly present the modal logic of context of [9]. Section 3 is devoted to extend this logic with the two events $X+\psi$ and $X-\psi$ which allow to model context dynamics. Finally, in Section 4 we will apply our logical framework to norm change, i.e. norm promulgation and norm derogation. The results of this application are compared with related work in Section 5 . Conclusions follow.

\section{A Modal Logic of Context}

The logic presented in this section is a simple modal logic designed to represent and reason about a localized notion of validity, that is, of validity with respect to all models in a given set. Such a given set is what is here called a context.

Let $\Phi=\{p, q, \ldots\}$ be a countable non-empty set of propositional letters, and let $\mathcal{C}=\{X, Y, \ldots\}$ be a countable set of contexts. $\mathcal{L}_{\text {Prop }}$ is the propositional language.

\subsection{Models}

Definition 1. A context model (Cxt-model) $\mathcal{M}=(W, R, \mathcal{I})$ is a tuple such that:

- $W$ is a nonempty set of possible worlds;

- $R: \mathcal{C} \longrightarrow 2^{W}$ maps each context $X$ to a subset of $W$;

- $\mathcal{I}: \Phi \longrightarrow 2^{W}$ is a valuation.

We write $R_{X}$ for $R(X)$ and $w \in \mathcal{M}$ for $w \in W$. For $w \in \mathcal{M}$, the couple $(\mathcal{M}, w)$ is a pointed context model.

A Cxt-model represents a logical space together with some of its possible restrictions, i.e., the contexts. In our case, contexts are used to represent the restrictions to those sets of propositional models satisfying the rules stated by a given normative system [9]. Let us illustrate how they can be used to model normative systems.

Example 1 (A toy normative system). Consider a normative system according to which: motorized vehicles must have a numberplate ; motorized vehicles must have an insurance; bikes should not have an insurance; bikes are classified as not being a motorized vehicle. Once a designated atom $\mathrm{V}$ is introduced in the language, which represents a notion of "violation" [4], the statements above obtain a simple representation:

Rule 1: $(m t \wedge \neg p l) \rightarrow \mathrm{V}$ Rule 2: $(m t \wedge \neg i n) \rightarrow \mathrm{V}$

Rule 3: $(b k \wedge i n) \rightarrow \bigvee$ Rule 4: $\quad b k \rightarrow \neg m t$

A Cxt-model $\mathcal{M}=(W, R, \mathcal{I})$, where $\mathcal{I}$ maps atoms $m t, p l, i n, b k$ and $\mathrm{V}$ to subsets of $W$, models the normative system above as a context $X$ if $R_{X}$ coincides with the subset of $W$ where Rules 1-4 are true according to propositional logic. 


\subsection{Logic}

The logic Cxt is now presented which captures the notion of validity with respect to a context. To talk about $\mathbf{C x t}$-models we use a modal language $\mathcal{L}_{\mathrm{Cxt}}$ containing modal operators $[X]$ for every $X \in \mathcal{C}$, plus the universal modal operator $[\mathrm{U}]$. The set of well-formed formulae of $\mathcal{L}_{\mathrm{Cxt}}$ is defined by the following BNF:

$$
\mathcal{L}_{\mathrm{Cxt}}: \varphi::=p|\neg \varphi| \varphi \wedge \varphi|[\mathrm{U}] \varphi|[X] \varphi
$$

where $p$ ranges over $\Phi$ and $X$ over $\mathcal{C}$. The connectives $\top, \vee, \rightarrow, \leftrightarrow$ and the dual operators $\langle X\rangle$ are defined as usual within $\mathcal{L}_{\mathrm{Cxt}}$ as: $\langle X\rangle \varphi=\neg[X] \neg \varphi$, for $X \in \mathcal{C} \cup\{\mathrm{U}\}$.

We interpret formulas of $\mathcal{L}_{\mathrm{Cxt}}$ in a Cxt-models as follows: the $[\mathrm{U}]$ operator is interpreted as the universal modality [5], and the $[X]$ operators model restricted validity.

Definition 2. Let $\mathcal{M}$ be a Cxt-model, and let $w \in \mathcal{M}$.

$\mathcal{M}, w \models p$ iff $w \in \mathcal{I}(p)$;

$\mathcal{M}, w \models[X] \varphi$ iff for all $w^{\prime} \in R_{X}, \mathcal{M}, w^{\prime} \models \varphi ;$

$\mathcal{M}, w \models[\mathrm{U}] \varphi$ iff for all $w^{\prime} \in W, \mathcal{M}, w^{\prime} \models \varphi$.

and as usual for the Boolean operators. Formula $\varphi$ is valid in $\mathcal{M}$, noted $\mathcal{M} \models \varphi$, iff $\mathcal{M}, w \models \varphi$ for all $w \in \mathcal{M} . \varphi$ is Cxt-valid, noted $\models{ }_{\mathbf{C x t}} \varphi$, iff $\mathcal{M} \models \varphi$ for all Cxt-models $\mathcal{M}$.

Cxt-validity is axiomatized by the following schemas:

(P) all propositional axiom schemas and rules

$$
\begin{aligned}
& \left(4^{X Y}\right)[X] \varphi \rightarrow[Y][X] \varphi \\
& \left(5^{X Y}\right)\langle X\rangle \varphi \rightarrow[Y]\langle X\rangle \varphi \\
& \left(\mathrm{T}^{\mathrm{U}}\right)[\mathrm{U}] \varphi \rightarrow \varphi \\
& \left(\mathrm{K}^{X}\right)[X]\left(\varphi \rightarrow \varphi^{\prime}\right) \rightarrow\left([X] \varphi \rightarrow[X] \varphi^{\prime}\right) \\
& \left(\mathrm{N}^{X}\right) \quad \mathrm{IF} \vdash \varphi \text { THEN } \vdash[X] \varphi
\end{aligned}
$$

where $X, Y \in \mathcal{C} \cup\{\mathrm{U}\}$. The $[X]$ and $[Y]$ operators are $\mathbf{K} 45$ modalities strengthened with the two inter-contextual interaction axioms $4^{X Y}$ and $5^{X Y}$. [U] is an $\mathbf{S 5}$ modality. Provability of a formula $\varphi$, noted $\vdash_{\mathbf{C x t}} \varphi$, is defined as usual.

Logic Cxt is well-behaved for both axiomatizability and complexity.

Theorem 1 ([9]). $\models_{\mathbf{C x t}} \varphi$ iff $\vdash_{\mathbf{C x t}} \varphi$.

Theorem 2. Deciding Cxt-validity is coNP-complete.

Proof (Sketch of proof). Satisfiability of $\mathbf{S 5}$ formulas is decidable in nondeterministic polynomial time [5]. Let $\mathcal{L}^{[\mathrm{U}]}$ be the language built from the set of atoms $\Phi \cup \mathcal{C}$ (supposing $\Phi$ and $\mathcal{C}$ are disjoint) and containing only one modal operator [U]. That is:

$$
\mathcal{L}^{[\mathrm{U}]}: \varphi::=p|\neg \varphi| \varphi \wedge \varphi \mid[\mathrm{U}] \varphi
$$

where $p$ ranges over $\Phi \cup \mathcal{C}$. It gets a natural interpretation on context models where $[\mathrm{U}]$ is the global modality. Then one can show that the following is a satisfiability-preserving polytime reduction $f$ of $\mathcal{L}_{\mathrm{Cxt}}$ to $\mathcal{L}^{[\mathrm{U}]}: f(p)=p ; f(\neg \varphi)=\neg f(\varphi) ; f\left(\varphi \wedge \varphi^{\prime}\right)=$ $f(\varphi) \wedge f\left(\varphi^{\prime}\right) ; f([\mathrm{U}] \varphi)=[\mathrm{U}] f(\varphi) ; f([X] \varphi)=[\mathrm{U}](X \rightarrow f(\varphi))$.

The same argument proves linear time complexity if the alphabet $\Phi$ is finite. 
Another interesting property of $\mathbf{C x t}$ is that every formula of $\mathcal{L}_{\mathbf{C x t}}$ is provably equivalent to a formula without nested modalities, as the following proposition shows. We first formally define the language without nested modalities:

$$
\mathcal{L}_{\mathbf{C x t}}^{1}: \varphi::=\alpha|[X] \alpha|[\mathrm{U}] \alpha|\neg \varphi| \varphi \wedge \varphi
$$

where $\alpha$ ranges over $\mathcal{L}_{\text {Prop }}$ and $X$ over $\mathcal{C}$. This result is of use in Proposition 3 .

Proposition 1. For all $\varphi \in \mathcal{L}_{\mathbf{C x t}}$ there is $\varphi^{1} \in \mathcal{L}_{\mathbf{C x t}}^{1}$ such that $\vdash_{\mathbf{C x t}} \varphi \leftrightarrow \varphi^{1}$.

Proof. By induction on $\varphi$. The Boolean cases clearly work. If $\varphi$ is of the form $[X] \psi$ with $X \in \mathcal{C} \cup\{\mathrm{U}\}$ then by IH there are $\alpha_{k}, \alpha_{j}^{i}, \beta^{i} \in \mathcal{L}_{\text {Prop }}$ such that

$$
\left.\varphi \leftrightarrow[X] \bigwedge_{k \in \mathbb{N}_{l}}\left(\alpha_{k} \vee \bigvee_{i \in \mathbb{N}_{n_{k}}}\left(\left[X_{i}\right] \alpha_{1}^{i} \vee \ldots \vee\left[X_{i}\right] \alpha_{n_{i}}^{i} \vee\left\langle X_{i}\right\rangle \beta^{i}\right)\right)\right)
$$

However, using $\left(4^{X Y}\right)$ and $\left(5^{X Y}\right)$, one can easily show that

$$
\begin{gathered}
\left.\vdash_{\mathbf{C x t}}[X]\left(\alpha_{k} \vee \bigvee_{i \in \mathbb{N}_{n_{k}}}\left(\left[X_{i}\right] \alpha_{1}^{i} \vee \ldots \vee\left[X_{i}\right] \alpha_{n_{i}}^{i} \vee\left\langle X_{i}\right\rangle \beta^{i}\right)\right)\right) \leftrightarrow \\
\left.\left([X] \alpha_{k} \vee \bigvee_{i \in \mathbb{N}_{n_{k}}}\left(\left[X_{i}\right] \alpha_{1}^{i} \vee \ldots \vee\left[X_{i}\right] \alpha_{n_{i}}^{i} \vee\left\langle X_{i}\right\rangle \beta^{i}\right)\right)\right) .
\end{gathered}
$$

This completes the proof.

\subsection{Normative Systems in Cxt}

We are ready to provide an object-level representation of Example 1. The contextual operators $[X]$ and the universal operator $[\mathrm{U}]$ can be used to define the concepts of classificatory rule, obligation and permission which are needed to model normative systems. Classificatory rules are of the form " $\varphi$ counts as $\psi$ in the normative system $X$ " and their function in a normative systems is to specify classifications between different concepts [15]. For example, according to the classificatory rule "in the context of Europe, a piece of paper with a certain shape, color, etc. counts as a 5 Euro bill", in Europe a piece of paper with a certain shape, color, etc. should be classified as a 5 Euro bill. The concept of classificatory rule is expressed by the following abbreviation:

$$
\varphi \Rightarrow_{X} \psi \stackrel{\text { def }}{=}[X](\varphi \rightarrow \psi)
$$

where $\varphi \Rightarrow_{X} \psi$ reads ' $\varphi$ counts as $\psi$ in normative system $X$ '. As done already in Example 1, by introducing the violation atom $\mathrm{V}$ we can obtain a reduction of deontic logic to logic $\mathbf{C x t}$ along the lines first explored by Anderson [4]. As far as obligations are concerned, we introduce operators of the form $\mathbf{O}_{X}$ which are used to specify what is obligatory in the context of a certain normative system $X$ :

$$
\mathbf{O}_{X} \varphi \stackrel{\text { def }}{=} \neg \varphi \Rightarrow_{X} \mathrm{~V}
$$

According to this definition, ' $\varphi$ is obligatory within context $X$ ' is identified with ' $\neg \varphi$ counts as a violation in normative system $X^{\prime}$. Note that we have the following Cxt-theorem: 


$$
\vdash_{\mathbf{C x t}}\left(\left(\varphi \Rightarrow_{X} \psi\right) \wedge\left(\varphi \Rightarrow_{X} \neg \psi\right)\right) \rightarrow \mathbf{O}_{X} \neg \varphi
$$

Every $\mathbf{O}_{X}$ obeys axiom $\mathrm{K}$ and necessitation, and is therefore a normal modal operator.

$$
\begin{aligned}
& \vdash_{\mathbf{C x t}} \quad \mathbf{O}_{X}(\varphi \rightarrow \psi) \rightarrow\left(\mathbf{O}_{X} \varphi \rightarrow \mathbf{O}_{X} \psi\right) \\
& \text { IF } \vdash_{\mathbf{C x t}} \varphi \text { THEN } \vdash_{\mathbf{C x t}} \mathbf{O}_{X} \varphi
\end{aligned}
$$

Note that the formula $\mathbf{O}_{X} \perp$ is consistent, hence our deontic operator does not satisfy the $\mathrm{D}$ axiom.

We define the permission operator in the standard way as the dual of the obligation operator: " $\varphi$ is permitted within context $X$ ", noted $\mathbf{P}_{X} \varphi$. Formally:

$$
\mathbf{P}_{X} \varphi \stackrel{\text { def }}{=} \neg \mathbf{O}_{X} \neg \varphi
$$

Formula $\mathbf{P}_{\cup} \varphi$ should be read " $\varphi$ is deontically possible".

Example 2 (Talking about a toy normative system). Consider again the normative system of Example 1. We can now express in Cxt that Rules 1-4 explicitly belong to context $X$ :

Rule 1: $\mathbf{O}_{X}(m t \rightarrow p l)$ Rule 2: $\mathbf{O}_{X}(m t \rightarrow i n)$

Rule 3: $\mathbf{O}_{X}(b k \rightarrow \neg i n)$ Rule 4: $b k \Rightarrow_{X} \neg m t$

Rules $1^{\prime}-4^{\prime}$ explicitly localize the validity of Rules 1-4 of Example 1 to context $X$. Logic Cxt is therefore enough expressive to represent several (possibly inconsistent) normative systems at the same time.

\section{Dynamic Context Logic}

In the present section we 'dynamify' logic Cxt.

\subsection{Two Relations on Models}

We first define the relations $\stackrel{X+\psi}{\longrightarrow}$ and $\stackrel{X-\psi}{\longrightarrow}$ on the set of pointed Cxt-models.

Definition 3. Let $(\mathcal{M}, w)=(W, R, \mathcal{I}, w)$ and $\left(\mathcal{M}^{\prime}, w^{\prime}\right)=\left(W^{\prime}, R^{\prime}, \mathcal{I}^{\prime}, w^{\prime}\right)$ be two pointed Cxt-models, and let $\varphi \in \mathcal{L}_{\mathbf{C x t}}$ and $X \in \mathcal{C}$.

We $\operatorname{set}(\mathcal{M}, w) \stackrel{X+\psi}{\longrightarrow}\left(\mathcal{M}^{\prime}, w^{\prime}\right)$ iff $W=W^{\prime}, w=w^{\prime}, \mathcal{I}=\mathcal{I}^{\prime}$, and

$-R_{Y}^{\prime}=R_{Y}$ if $Y \neq X$;

- $R_{X}^{\prime}=R_{X} \cap\|\psi\|_{\mathcal{M}}$.

We $\operatorname{set}(\mathcal{M}, w) \stackrel{X-\psi}{\longrightarrow}\left(\mathcal{M}^{\prime}, w^{\prime}\right)$ iff $W=W^{\prime}, w=w^{\prime}, \mathcal{I}=\mathcal{I}^{\prime}$, and

- $R_{Y}^{\prime}=R_{Y}$ if $Y \neq X$;

- $R_{X}^{\prime}=\left\{\begin{array}{l}R_{X} \text { if } \mathcal{M}, w \models \neg[X] \psi \vee[U] \psi \\ R_{X} \cup S \text { otherwise, for some } \emptyset \neq S \subseteq\|\neg \psi\|_{\mathcal{M}}\end{array}\right.$

In case $(\mathcal{M}, w) \stackrel{X+\psi}{\longrightarrow}\left(\mathcal{M}^{\prime}, w^{\prime}\right)$ (resp. $\left.(\mathcal{M}, w) \stackrel{X-\psi}{\longrightarrow}\left(\mathcal{M}^{\prime}, w^{\prime}\right)\right)$, we say that $\mathcal{M}^{\prime}$ is a (context) expansion (resp. contraction) of $\mathcal{M}$. 
In the above definition, $\|\psi\|_{\mathcal{M}}=\{w \in \mathcal{M}: \mathcal{M}, w \mid=\psi\}$. So in both cases, it is only the context $X$ which changes from $\mathcal{M}$ to $\mathcal{M}^{\prime}$. In the first case, it is restricted to the worlds that satisfy $\psi$, and in the second case, it is enlarged with some worlds which satisfy $\neg \psi$, except if such worlds do not exist in the model ([U] $\psi$ ) or if $\neg \varphi$ is already consistent with the context $(\neg[X] \psi)$. Note that there might be several contractions of a given Cxt-model but there is always a unique expansion. The relation $\stackrel{X-\psi}{\longrightarrow}$ thus defines implicitly a family of contraction operations. The following proposition shows that $\stackrel{X-\psi}{\longrightarrow}$ is essentially the converse relation of $\stackrel{X+\psi}{\longrightarrow}$.

Proposition 2. Let $(\mathcal{M}, w)$ and $\left(\mathcal{M}^{\prime}, w^{\prime}\right)$ be two pointed $\mathbf{C x t}$-models and $\psi \in \mathcal{L}_{\mathbf{C x t}}$. Then $(\mathcal{M}, w) \stackrel{X+\psi}{\longrightarrow}\left(\mathcal{M}^{\prime}, w^{\prime}\right)$ iff $\left(\mathcal{M}^{\prime}, w^{\prime}\right) \stackrel{X-\psi}{\longrightarrow}(\mathcal{M}, w)$ and $\mathcal{M}^{\prime}, w^{\prime} \models[X] \psi$.

Proof. The left to right direction is clear. Assume that $\left(\mathcal{M}^{\prime}, w^{\prime}\right) \stackrel{X-\psi}{\longrightarrow}(\mathcal{M}, w)$ and $\mathcal{M}^{\prime}, w^{\prime} \models[X] \psi$. Then $R_{Y}^{\prime}=R_{Y}$ if $Y \neq X$ by definition. If $\mathcal{M}^{\prime}, w^{\prime} \models \neg[\mathrm{U}] \psi$ then $R_{X}^{\prime}=R_{X} \cup S$ for some $\emptyset \neq S \subseteq\|\neg \psi\|_{\mathcal{M}}$ because $\mathcal{M}^{\prime}, w^{\prime} \models[X] \psi \wedge \neg[\mathrm{U}] \psi$. So $R_{X}^{\prime}=R_{X} \cap\|\psi\|_{\mathcal{M}}$. Otherwise, if $\mathcal{M}^{\prime}, w^{\prime}=[\mathrm{U}] \psi$ then $R_{X}^{\prime}=R_{X}$ by definition. So $R_{X}^{\prime}=R_{X} \cap\|\psi\|_{\mathcal{M}}$ because $\mathcal{M}^{\prime}, w^{\prime}=[X] \psi$. In both cases $R_{X}^{\prime}=R_{X} \cap\|\psi\|_{\mathcal{M}}$. Therefore $(\mathcal{M}, w) \stackrel{X+\psi}{\longrightarrow}\left(\mathcal{M}^{\prime}, w^{\prime}\right)$.

\subsection{Logic}

The language of the logic DCxt is obtained by adding the dynamic operators $[X+\psi]$ and $[X-\psi]$ to the language $\mathcal{L}_{\mathbf{C x t}}$ :

$$
\mathcal{L}_{\text {DCxt }}: \varphi::=p|\neg \varphi| \varphi \wedge \varphi|[X] \varphi|[\mathrm{U}] \varphi|[X+\psi] \varphi|[X-\psi] \varphi
$$

where $p$ ranges over $\Phi, X$ over $\mathcal{C}$ and $\psi$ over $\mathcal{L}_{\mathbf{C x t}} \cdot[X+\psi] \varphi$ reads 'after the expansion of the context $X$ by $\psi, \varphi$ is true', and $[X-\psi] \varphi$ reads 'after any contraction of the context $X$ by $\psi, \varphi$ is true'.

Definition 4. Let $\mathcal{M}$ be a $\mathbf{C x t}$-model. The truth conditions for $\mathcal{L}_{\mathrm{DCxt}}$ in $\mathcal{M}$ are those of Definition 2 plus:

$$
\begin{aligned}
& \mathcal{M}, w \models[X+\psi] \varphi \text { iff } \mathcal{M}^{\prime}, w^{\prime} \models \varphi \text { for all Cxt-models }\left(\mathcal{M}^{\prime}, w^{\prime}\right) \\
& \text { such that }(\mathcal{M}, w) \stackrel{X+\psi}{\longrightarrow}\left(\mathcal{M}^{\prime}, w^{\prime}\right) \text {; } \\
& \mathcal{M}, w \models[X-\psi] \varphi \text { iff } \mathcal{M}^{\prime}, w^{\prime} \models \varphi \text { for all Cxt-models }\left(\mathcal{M}^{\prime}, w^{\prime}\right) \\
& \text { such that }(\mathcal{M}, w) \stackrel{X-\psi}{\longrightarrow}\left(\mathcal{M}^{\prime}, w^{\prime}\right) \text {. }
\end{aligned}
$$

As before, $\mathcal{M} \models \varphi$ iff $\mathcal{M}, w \models \varphi$ for all $w \in \mathcal{M}$, and $\varphi$ is DCxt-valid $\left(\mid={ }_{\operatorname{DCxt}} \varphi\right)$ iff $\mathcal{M} \models \varphi$ for all $\mathbf{C x t}$-models $\mathcal{M}$.

The operator $[X-\psi]$ is thus useful if we want to have general properties about our family of contractions or about a situation; for example, given some formulas $\psi_{1}, \ldots, \psi_{n}$, what would be true after any sequence of contractions and expansions by these formulas? Can we get an inconsistency with a specific choice of contractions? 
In order to axiomatize the DCxt-validities we define for every $X \in \mathcal{C}$ two auxiliary languages $\mathcal{L}_{\neq X}$ and $\mathcal{L}_{=X}$ :

$$
\begin{aligned}
& \mathcal{L}_{=X}: \varphi::=[X] \alpha|\neg \varphi| \varphi \wedge \varphi \\
& \mathcal{L}_{\neq X}: \varphi::=\alpha|[Y] \alpha| \neg \varphi \mid \varphi \wedge \varphi
\end{aligned}
$$

where $\alpha$ ranges over $\mathcal{L}_{\text {Prop }}$ and $Y$ over $(\mathcal{C} \cup\{\mathrm{U}\})-\{X\}$.

Logic DCxt is axiomatized by the following schemata:

(Cxt) All axiom schemas and inference rules of $\mathbf{C x t}$

$(\mathrm{R}+1)[X+\psi] \varphi_{\neq X} \leftrightarrow \varphi_{\neq X}$

$(\mathrm{R}+2)[X+\psi][X] \alpha \leftrightarrow[X](\psi \rightarrow \alpha)$

$(\mathrm{R}+3)[X+\psi] \neg \varphi \leftrightarrow \neg[X+\psi] \varphi$

$(\mathrm{R}-1)[X-\psi]\left(\varphi_{\neq X} \vee \varphi=X\right) \leftrightarrow\left(\varphi \neq X \vee[X-\psi] \varphi_{X}\right)$

$(\mathrm{R}-2) \neg[X-\psi] \perp$

$(\mathrm{R}-3)[X-\psi]\left([X] \alpha_{1} \vee \ldots \vee[X] \alpha_{n} \vee\langle X\rangle \alpha\right) \leftrightarrow$

$\left((\neg[X] \psi \vee[\mathrm{U}] \psi) \wedge\left([X] \alpha_{1} \vee \ldots \vee[X] \alpha_{n} \vee\langle X\rangle \alpha\right)\right)$

$\vee(([X] \psi \wedge \neg[\mathrm{U}] \psi) \wedge$

$\left.\left(\left(\bigvee_{i}\left([X] \alpha_{i} \wedge[\mathrm{U}]\left(\psi \vee \alpha_{i}\right)\right)\right) \vee\langle X\rangle \alpha \vee[\mathrm{U}](\psi \vee \alpha)\right)\right)$

$\left(\mathrm{K}^{+}\right)[X+\psi]\left(\varphi \rightarrow \varphi^{\prime}\right) \rightarrow\left([X+\psi] \varphi \rightarrow[X+\psi] \varphi^{\prime}\right)$

$\left(\mathrm{K}^{-}\right)[X-\psi]\left(\varphi \rightarrow \varphi^{\prime}\right) \rightarrow\left([X-\psi] \varphi \rightarrow[X-\psi] \varphi^{\prime}\right)$

(RRE) Rule of replacement of proved equivalence

where $X \in \mathcal{C}, \varphi, \varphi^{\prime} \in \mathcal{L}_{\mathbf{D C x t}}, \psi \in \mathcal{L}_{\mathbf{C x t}}, \varphi=X \in \mathcal{L}_{=X}, \varphi \neq X \in \mathcal{L}_{\neq X}$, and $\alpha, \alpha_{i} \ldots \in$ $\mathcal{L}_{\text {Prop }}$.

Note that from $(\mathrm{R}-1)$ and $(\mathrm{R}-2)$ one can deduce $[X-\psi] \varphi_{\neq X} \leftrightarrow \varphi_{\neq X}$. The formulae above are reduction axioms:

Proposition 3. For all $\varphi_{\mathbf{D C x t}} \in \mathcal{L}_{\mathbf{D C x t}}$ there is $\varphi_{\mathbf{C x t}} \in \mathcal{L}_{\mathbf{C x t}}$ such that $\vdash_{\mathbf{D C x t}}$ $\varphi_{\text {DCxt }} \leftrightarrow \varphi_{\text {Cxt }}$.

Proof (Sketch of proof). (By induction on the number of occurrences of dynamic operators.) Let $\varphi_{\text {DCxt }} \in \mathcal{L}_{\text {DCxt }}$ and $\varphi_{\text {DCxt }}^{\prime}$ be one of its sub-formulas of the form $[X+\psi] \varphi_{\mathbf{C x t}}$ or $[X-\psi] \varphi_{\mathbf{C x t}}$, with $\varphi_{\mathbf{C x t}} \in \mathcal{L}_{\mathbf{C x t}}$. By Proposition 1, there is $\varphi_{\mathbf{C x t}}^{1} \in$ $\mathcal{L}_{\mathbf{C x t}}^{1}$ such that $\vdash_{\mathbf{C x t}} \varphi_{\mathbf{C x t}} \leftrightarrow \varphi_{\mathbf{C x t}}^{1}$. So $\vdash_{\text {DCxt }}[X+\psi] \varphi_{\mathbf{C x t}} \leftrightarrow[X+\psi] \varphi_{\mathbf{C x t}}^{1}$ by $(\mathrm{REE})$ and $\left(K^{+}\right)$. Now, thanks to axioms $(\mathrm{R}+1),(\mathrm{R}+2)$ and $(\mathrm{R}+3)$ and because $\varphi_{\mathbf{C x t}}^{1} \in \mathcal{L}_{\mathbf{C x t}}^{1}$, one can easily show that there is $\psi_{\mathbf{C x t}} \in \mathcal{L}_{\mathbf{C x t}}$ such that $\vdash_{\mathbf{D C x t}}[X+\psi] \varphi_{\mathbf{C x t}}^{1} \leftrightarrow \psi_{\mathbf{C x t}}$. For the case $[X-\psi] \varphi_{\text {Cxt }}$ we apply the same method using $(\mathrm{R}-1),(\mathrm{R}-2)$ and $(\mathrm{R}-3)$. So $\vdash_{\text {DCxt }} \varphi_{\text {DCxt }}^{\prime} \leftrightarrow \psi_{\text {Cxt }}$. Now we replace $\varphi_{\text {DCxt }}^{\prime}$ by $\psi_{\text {Cxt }}$ in $\varphi_{\text {DCxt }}$. This yields an equivalent formula (thanks to ( $R R E)$ ) with one dynamic operator less. We then apply to this formula the same process we applied to $\varphi_{\mathbf{C x t}}$ until we get rid of all the dynamic operators. 
So, if we want to check that a given formula of the form $\left[X \pm \psi_{1}\right] \ldots\left[X \pm \psi_{n}\right] \varphi$ holds in a Cxt-model, instead of computing all the corresponding sequences of contractions and expansions $\psi_{1}, \ldots, \psi_{n}$ of the $\mathbf{C x t}$-model, we can also reduce the formula to one of $\mathcal{L}_{\mathbf{C x t}}$ and check it on the original Cxt-model. This way to proceed might be computationally less costly. For example, $\vdash_{\text {DCxt }}[X-\alpha] \neg[X] \alpha \leftrightarrow\langle\mathrm{U}\rangle \neg \alpha$. As in DEL, soundness, completeness and decidability follow from Proposition 3

\section{Theorem 3. $\models$ DCxt $\varphi$ iff $\vdash_{\text {DCxt }} \varphi$. Deciding DCxt-validity is decidable.}

Finally, it should be noted that we could easily enrich this formalism with specific contraction operators. For example we could add to $\mathcal{L}_{\mathbf{D C x t}}$ the contraction operator $[X \stackrel{\circ}{=}] \varphi$ whose semantics would be defined as follows: for $\mathcal{M}=(W, R, \mathcal{I}), \mathcal{M}, w \models$ $[X \stackrel{\circ}{=}] \varphi$ iff $\mathcal{M}^{\prime}, w \models \varphi$, where $\mathcal{M}^{\prime}=\left(W, R^{\prime}, \mathcal{I}\right)$ with $R_{Y}^{\prime}=R_{Y}$ for $Y \neq X$ and $R_{X}^{\prime}=R_{X} \cup\{w \in W \mid \mathcal{M}, w \models \neg \psi\}$. To get a complete axiomatization, we just

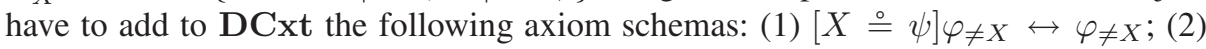

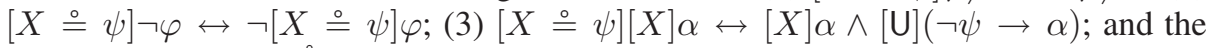
distribution axiom $\left(\mathrm{K}^{\circ}\right)$. In fact this contraction $\stackrel{\circ}{=}$ belongs to the family of contractions defined in Definition 3 and so we get $\vdash_{\text {DCxt }}[X-\psi] \varphi \rightarrow[X \stackrel{\circ}{=} \psi]$.

\section{A Logical Account of Norm Change}

Just as we defined the static notions of obligation and classificatory rules on the basis of $\mathbf{C x t}$, we can in the same spirit define the dynamic notions of promulgation and derogation of obligation and classificatory rules on the basis of DCxt:

$$
\begin{aligned}
& +\left(\varphi \Rightarrow_{X} \psi\right) \stackrel{\text { def }}{=} X+(\varphi \rightarrow \psi)+\mathrm{O}_{X} \psi \stackrel{\text { def }}{=} X+(\neg \psi \rightarrow \mathrm{V}) \\
& -\left(\varphi \Rightarrow_{X} \psi\right) \stackrel{\text { def }}{=} X-(\varphi \rightarrow \psi)-\mathrm{O}_{X} \psi \stackrel{\text { def }}{=} X-(\neg \psi \rightarrow \mathrm{V})
\end{aligned}
$$

Operator $\left[H\left(\varphi \Rightarrow_{X} \psi\right)\right] \chi$ (resp. $\left.\left[-\left(\varphi \Rightarrow_{X} \psi\right)\right] \chi\right)$ should be read 'after the promulgation (resp. after any derogation) of the classificatory rule $\varphi \Rightarrow_{X} \psi, \chi$ is true'. Likewise, $\left[+\mathbf{O}_{X} \psi\right] \varphi$ (resp. $\left[-\mathbf{O}_{X} \psi\right] \varphi$ ) should be read 'after the promulgation (resp. after any derogation) within context $X$ of the obligation $\psi, \chi$ is true'.

Example 3 (Changing a toy normative system). In Example 2] after the legislator's proclamation that motorized vehicles having more than 50cc $(\mathrm{mf})$ are obliged to have a numberplate (event $+\mathbf{O}_{X}((m t \wedge m f) \rightarrow p l)$ and that motorized vehicles having less than 50cc $(\neg m f)$ are not obliged to have a numberplate (event $-\mathrm{O}_{X}((m t \wedge \neg m f) \rightarrow$ $p l$ ) we should expect that motorbikes having more than $50 \mathrm{cc}$ have the obligation to have a numberplate and motorbikes having less than 50cc have the permission not to have a numberplate. This is indeed the case as the following formula is a theorem:

$$
\begin{aligned}
& \mathbf{P}_{\mathrm{U}}(m t \wedge \neg m f \wedge \neg p l) \rightarrow\left(\left[+\mathbf{O}_{X}((m t \wedge m f) \rightarrow p l)\right]\left[-\mathbf{O}_{X}((m t \wedge \neg m f) \rightarrow p l)\right]\right. \\
& \left.\mathbf{O}_{X}((m t \wedge m f) \rightarrow p l) \wedge \mathbf{P}_{X}(m t \wedge \neg m f \wedge \neg p l)\right) .
\end{aligned}
$$

More generally, we have the following proposition. 
Proposition 4. The following formulae are $\mathrm{DCxt}$-theorems:

$$
\begin{aligned}
& {\left[H\left(\varphi \Rightarrow_{X} \psi\right)\right] \varphi \Rightarrow_{X} \psi} \\
& {\left[+\mathbf{O}_{X} \psi\right]_{X} \psi} \\
& \mathbf{P}_{U} \neg \psi \rightarrow\left[-\mathbf{O}_{X} \psi\right] \mathbf{P}_{X} \neg \psi \\
& \left(\left(\varphi \Rightarrow_{X} \psi\right) \wedge\langle\mathrm{U}\rangle \neg(\varphi \rightarrow \psi)\right) \rightarrow \\
& {\left[-\left(\varphi \Rightarrow_{X} \psi\right)\right]\left[H\left(\varphi \Rightarrow_{X} \neg \psi\right)\right] \neg\left(\left(\varphi \Rightarrow_{X} \neg \psi\right) \wedge\left(\varphi \Rightarrow_{X} \psi\right)\right)} \\
& \left(\mathbf{O}_{X}(\varphi \rightarrow \psi) \wedge \mathbf{P}_{U} \neg(\varphi \rightarrow \psi)\right) \rightarrow \\
& {\left[-\mathbf{O}_{X}(\varphi \rightarrow \psi)\right]\left[+\mathbf{O}_{X}(\varphi \rightarrow \neg \psi)\right]\left(\mathbf{O}_{X}(\varphi \rightarrow \neg \psi) \wedge \mathbf{O}_{X}(\varphi \rightarrow \psi)\right)} \\
& \left(\mathbf{P}_{U} \neg(\psi \rightarrow \varphi) \wedge \mathbf{O}_{X} \varphi\right) \rightarrow\left[-\mathbf{O}_{X}(\psi \rightarrow \varphi)\right] \neg \mathbf{O}_{X} \varphi \\
& \left(\varphi \Rightarrow_{X} \psi\right) \rightarrow\left(\left[-\mathbf{O}_{X} \varphi\right] \xi \rightarrow\left[-\mathbf{O}_{X} \psi\right] \xi\right) \\
& \left(\langle\mathrm{U}\rangle \neg(\varphi \rightarrow \psi) \wedge\left(\varphi \Rightarrow{ }_{X} \psi\right) \wedge\left(\psi \Rightarrow_{X} \xi\right)\right) \rightarrow\left\langle-\varphi \Rightarrow_{X} \psi\right\rangle \neg\left(\varphi \Rightarrow_{X} \xi\right) \\
& \neg[X] \psi \rightarrow(\varphi \leftrightarrow[X-\psi] \varphi) \\
& \alpha \rightarrow[X-\psi][X+\psi] \alpha \quad \text { for } \alpha \in \mathcal{L}_{P r o p} \\
& {[Y] \alpha \rightarrow[X-\psi][X+\psi][Y] \alpha \quad \text { for } \alpha \in \mathcal{L}_{\text {Prop }}}
\end{aligned}
$$

Proofs are omitted for space reasons but the theorems can easily be checked semantically. Let us spell out the intuitive readings of these formulae. Formulae 4 and 5 simply state the obvious consequences of the expansion of a context with a classificatory rule and with an obligation. Formula 6 states that if a state of affairs can possibly be permitted, then derogating the obligation for that state of affairs gives rise to a permission for that state of affairs. It is worth noticing that this captures a notion of "strong permission", as it is often called in the literature on deontic logic (see, for instance, [11]), that is, a permission which is obtained as the effect of an explicit derogation to norms in force. Formulae 8 and 9 describe recipes for appropriately updating contexts. For instance, Formula 9 roughly says that if I want to make $\neg \psi$ obligatory in $\varphi$-situations starting from a context where $\psi$ is instead obligatory, I have to first derogate this latter obligation and then promulgate the desired one if I do not want to end up in situations where both $\psi$ and $\neg \psi$ are obligatory. Formula 9 states that if $\varphi$ is obligatory, then by derogating that $\varphi$ is obligatory in $\psi$-situations, an exception is introduced so that $\varphi$ is not obligatory in an unconditional way any more. Formula 10 says that, in the presence of a classificatory rule, by derogating the obligatoriness of the antecedent of the rule, we obtain a derogation of the obligatoriness of its consequent too. Finally, Formula 11 states that if I have two interpolated classificatory rules, by derogating one of them I undercut the conclusion I could draw by transitivity before the derogation. Formulae 12,14 are reminiscent of AGM postulates. Formula 12 expresses a form of minimality criterion, while Formulae 13 and 14 state two recovery principles for formulae belonging to a restricted language.

\section{Related Work on Norm Change}

Formal models of norm change have been drawing attention since the seminal work of Alchourrón and Makinson on the logical structure of derogation in legal codes [3] which expanded into a more general investigation of the logic of theory change (alias 
belief change) [2]. In this section we position our work with respect to AGM and related approaches to norm change available in the literature.

The first thing to notice about AGM is that its models are about the contraction of $\mathcal{L}_{\text {Prop }}$-theories, and focus on minimal change. In contrast, we consider here a modal language $\mathcal{L}_{\mathbf{C x t}}$. Our contraction operator "-." allows to express properties about a family of contractions, which actually do not necessarily satisfy the AGM criteria of minimal change. However, as shown in Proposition 4 our operator enjoys a minimality criterion (Formula 12) and two forms of recovery (Formulae 13 and 14. With respect to recovery it should be noticed, on the other hand, that formula $\neg[X] p \rightarrow[X-p][X+p] \neg[X] p$ is instead invalid, and hence that Formulae 13 and 14 do not generalize to all formulae in $\mathcal{L}_{\text {Cxt }}$.

Recently, norm change has gained quite some attention in the multi-agent systems community. As it is often the case, two main methodological approaches are recognizable: on the one hand syntactic approaches-inspired by legal practice-where norm change is considered as an operation performed directly on the explicit provisions contained in the "code" of the normative system [6, 8], and on the other hand semantic approaches, which are inspired by the dynamic logic paradigm [21] and which look at norm change as some form of model-update. Our contribution clearly falls in the second group and for this very reason our logic can be used for the formal specification and verification of computational models of norm-based interaction. Our approach is in fact close in spirit to Segerberg's [16], who argued for an integration of AGM belief revision with Hintikka-like static logics of belief: we here do the same for 'Andersonian' deontic logic.

From the proposals belonging to this latter group, it is worth comparing our work in particular with the approach proposed in [14]. There, an extension of the dynamic logic of permission (DLP) of [20] with operations of granting or revoking a permission was proposed. They call DLP dyn $_{n}$ this DLP extension. Their operations are similar to our operations of norm promulgation and norm derogation. DLP is itself an extension of PDL (propositional dynamic logic) [10] where actions are used to label transitions from one state to another state in a model. The $\mathrm{DLP}_{d y n}$ operation of granting a permission just augments the number of permitted transitions in a model, whereas the operation of revoking a permission reduces the number of permitted transitions. However there are important differences between our approach and Pucella \& Weissman's. For us, normative systems are more basic than obligations and permissions, and the latter are defined from (and grounded on) the former. Moreover, dynamics of obligations and permissions are particular cases of normative system change (normative system expansion and contraction). Thus, we can safely argue that our approach is more general than Pucella \& Weissman's in which only dynamics of permissions are considered. It is also to be noted that, while in our approach classificatory rules and their dynamics are crucial concepts in normative change, in $\mathrm{DLP}_{d y n}$ they are not considered and even not expressible. In future work we will analyze the relationships between DLP $_{d y n}$ and our logic, and possibly a reduction of $\mathrm{DLP}_{d y n}$ to our logic DCxt.

While Pucella \& Weissman's revocation of permissions corresponds to public announcements in DEL, no DEL approaches have proposed the counterpart of their operation of granting permissions, alias contractions (with the exception of [19], but in the 
framework of a logic of preference). Arguably, the reason for it is that it is difficult to define contraction operations both preserving standard properties of epistemic models such as transitivity and Euclidianity and allowing for reduction axioms. This is made instead possible in DCxt by the intercontextual interaction axioms $4^{X Y}$ and $5^{X Y}$.

\section{Conclusions}

We have introduced and studied a dynamic logic accounting for context change, and have applied it to analyze several aspects of the dynamics of norms, viz. the dynamics of permissions, obligations and classificatory rules. Although the logic has been applied here only to provide a formal analysis of norm-change, it is clear that its range of application is much broader. Viewed in its generality, the logic is a logic of the dynamics of propositional theories, and as such, can be naturally applied to formal epistemology by studying theory-change, or to non-monotonic reasoning by studying how the context of an argumentation evolves during, for instance, a dialogue game. This kind of applications are future research.

Acknowledgments. Davide Grossi is supported by Nederlandse Organisatie voor Wetenschappelijk Onderzoek (VENI grant Nr. 639.021.816). Guillaume Aucher is supported by Fond National de la Recherche (Luxembourg). We also thank the anonymous reviewers for their comments.

\section{References}

1. Ågotnes, T., van der Hoek, W., Rodriguez-Aguilar, J.A., Sierra, C., Wooldridge, M.: On the logic of normative systems. In: Proc. of IJCAI 2007, pp. 1181-1186. AAAI Press, Menlo Park (2007)

2. Alchourrón, C., Gärdenfors, P., Makinson, D.: On the logic of theory change: Partial meet contraction and revision functions. J. of Symbolic Logic 50, 510-530 (1985)

3. Alchourrón, C., Makinson, D.: Hierarchies of regulations and their logic. In: Hilpinen, R. (ed.) Deontic Logic: Introductory and Systematic Readings. D. Reidel (1981)

4. Anderson, A.: A reduction of deontic logic to alethic modal logic. Mind 22, 100-103 (1958)

5. Blackburn, P., de Rijke, M., Venema, Y.: Modal Logic. Cambridge Univ. Press, Cambridge (2001)

6. Boella, G., Pigozzi, G., van der Torre, L.: Normative framework for normative system change. AAMAS (1), 169-176 (2009)

7. Ghidini, C., Giunchiglia, F.: Local models semantics, or contextual reasoning = locality + compatibility. Artificial Intelligence 127(2), 221-259 (2001)

8. Governatori, G., Rotolo, A.: Changing legal systems: abrogation and annulment (part I: revision of defeasible theories). In: van der Meyden, R., van der Torre, L. (eds.) DEON 2008. LNCS (LNAI), vol. 5076, pp. 3-18. Springer, Heidelberg (2008)

9. Grossi, D., Meyer, J.-J.C., Dignum, F.: The many faces of counts-as: A formal analysis of constitutive-rules. J. of Applied Logic 6(2), 192-217 (2008)

10. Harel, D., Kozen, D., Tiuryn, J.: Dynamic Logic. MIT Press, Cambridge (2000)

11. Hilpinen, R. (ed.): New Studies in Deontic Logic. Synthese Library Series. Reidel (1981)

12. Kanger, S.: New fondations for ethical theory. In: Hilpinen, R. (ed.) Deontic Logic: Introductory and Systematic Readings, pp. 36-58. Reidel Publishing Company (1971) 
13. Lomuscio, A., Sergot, M.: Deontic intepreted systems. Studia Logica 75, 63-92 (2003)

14. Pucella, R., Weissman, V.: Reasoning about dynamic policies. In: Walukiewicz, I. (ed.) FOSSACS 2004. LNCS, vol. 2987, pp. 453-467. Springer, Heidelberg (2004)

15. Searle, J.R.: Speech acts: An essay in the philosophy of language. Cambridge Univ. Press, Cambridge (1969)

16. Segerberg, K.: Two traditions in the logic of belief: bringing them together. In: Ohlbach, H.J., Reyle, U. (eds.) Logic, Language and Reasoning: essays in honour of Dov Gabbay. Kluwer, Dordrecht (1999)

17. Stalnaker, R.: On the representation of context. J. of Logic, Language, and Information 7, 3-19 (1998)

18. Ågotnes, T., Wiebe van der Hoek, W., Tennenholtz, M., Wooldridge, M.: Power in normative systems. In: Decker, P., Sichman, J., Sierra, C., Castelfranchi, C. (eds.) Proceedings of the Eighth International Conference on Autonomous Agents and Multiagent Systems (AAMAS 2009), pp. 145-152 (2009)

19. van Benthem, J., Liu, F.: Dynamic logic of preference upgrade. J. of Applied Non-Classical Logics 17(2), 157-182 (2007)

20. Van der Meyden, R.: The dynamic logic of permission. J. of Logic and Computation 6, 465479 (1996)

21. van Ditmarsch, H., van der Hoek, W., Kooi, B.: Dynamic Epistemic Logic. Synthese Library Series, vol. 337. Springer, Heidelberg (2007) 